\title{
Enquêtes françaises sur la sériciculture chinoise et leur influence, fin XVIIIe-fin XIXe siècles
}

French investigations of Chinese silk production and their impact, from the late seventeenth century to the end of the nineteenth century

\section{Mau Chuan-Hui}

\section{OpenEdition} Journals

Édition électronique

URL : http://journals.openedition.org/dht/1176

DOI : $10.4000 /$ dht. 1176

ISSN : 1775-4194

Éditeur :

Centre d'histoire des techniques et de l'environnement du Cnam (CDHTE-Cnam), Société des élèves du CDHTE-Cnam

\section{Édition imprimée}

Date de publication : 1 décembre 2007

Pagination : 24-36

ISBN : 978-2-9530779-0-2

ISSN : 0417-8726

Référence électronique

Mau Chuan-Hui, «Enquêtes françaises sur la sériciculture chinoise et leur influence, fin XVIIIe-fin XIXe siècles ", Documents pour l'histoire des techniques [En ligne], 14 | $2^{\mathrm{e}}$ semestre 2007, mis en ligne le 09 novembre 2010, consulté le 07 septembre 2020. URL : http://journals.openedition.org/dht/1176 ; DOI : https://doi.org/10.4000/dht.1176 


\title{
Enquêtes françaises sur la sériciculture chinoise et leur influence, fin $\mathrm{XVII}^{\mathrm{e}}$-fin $\mathrm{XIX}^{\mathrm{e}}$ siècles
}

\author{
Mau Chuan-Hui \\ Collège de France, EHESS
}

\begin{abstract}
Résumé
Les échanges entre la France et la Chine au siècle des Lumières ont attiré l'attention de nombreux historimodernes. Néanmoins, l'influence chinoise sur la sériciculture française au XVIII siècle a été négligée tant par I'historiographie française que chinoise. Le savoir chinois séricicole avait pourtant alimenté la recherche française sur la sériciculture pendant cette période décisive.

Dans la présente étude, nous reconstituerons cette histoire de la circulation des techniques séricicoles qui se fit d'abord de la Chine vers l'Europe pour revenir à la Chine avec des « retouches » européennes, toujours sur l'initiative des Français. La première et la seconde partie se concentrent sur les efforts français à la recherche des techniques chinoises, ce qui mena à la création de « l'école française de sériciculture » au cours de la première moitié du XIX $X^{e}$ siècle.

L'affaiblissement de la Chine après les guerres de l'opium et, en Europe, l'épidémie des maladies des vers à soie marquèrent un tournant. Suite aux succès rencontrés en France dans la lutte contre ces fléaux, des hommes d'affaires occidentaux voulurent imposer leurs techniques modernes dans la sériciculture chinoise. Cela ne se fit pas sans difficulté et s'inscrivit dans un contexte complexe de l'histoire chinoise et de ses relations délicates avec les puissances occidentales et japonaise de la fin du XIXe siècle.
\end{abstract}

Résumés et mots clés en anglais sont regroupés en fin de volume, accompagnés des mots clés français. Les mots suivis d'un astérisque figurent dans un lexique en fin d'article.

$L^{2}$ a sériciculture est une industrie qui repose sur la production de cocons. Avant que les techniques d'étouffage des chrysalides dans leurs cocons aient été mises au point, le tirage de la soie se faisait essentiellement dans les foyers d'éleveurs par peur de la sortie des papillons dix jours environ après la montée* des vers. Plusieurs espèces de vers produisent de la soie, mais seuls les bombyx produisaient une soie valant de I'or grâce à sa qualité fine, souple et brillante. En général, on entend par sériciculture l'élevage des bombyx et la culture du mûrier dont les feuilles constituent la nourriture essentielle de ces précieuses chenilles. C'est à la fin du XvII siècle, grâce aux jésuites de Chine, que les Français prirent connaissance de la soie produite par des vers sauvages. Cet élevage, susceptible d'augmenter la productivité nationale, les intéressait beaucoup, et plusieurs expériences d'élevage furent faites par des savants français, mais aucun résultat positif ne fut obtenu.

Si, à la fin du xve siècle, le tissage des soieries commença à s'établir en France, la sériciculture y était encore peu développée, bien qu'elle existât dans le midi de la France depuis la fin du xille siècle' ${ }^{1}$. Au début du $x v I^{e}$ siècle, les soyeux lyonnais réussirent à fabriquer des soieries à grande façonnée*, ce qui leur permit au siècle suivant de rivaliser avec leurs prédécesseurs - les Italiens. En revanche, la production de la soie laissait toujours à désirer. La France devait importer tous les ans une quantité considérable de soies étrangères, notamment celles du Piémont, qui étaient indispensables pour la confection des soieries de qualité. Cela était

1. Pour plus de détails, voir Le Roy Ladurie E., Les Paysans de Languedoc, Paris, Mouton/La Haye, 1966, t. I, p. 216. 
dû, d'une part, au niveau insuffisant des techniques du moulinage*, mais aussi à une qualité fort peu satisfaisante de la soie brute, sans parler de la médiocrité de la productivité. Tout en poursuivant leurs efforts de perfectionnement des techniques du tirage de la soie et du moulinage, de nombreux savants français se lancèrent dans la recherche de techniques séricicoles, dans l'espoir d'augmenter la productivité d'une soie de qualité supérieure.

Appréciant l'excellence des produits de la soie venant de l'Extrême-Orient, les Français s'intéressèrent au savoir chinois de la sériciculture et saisirent, à la fin du xvII siècle, l'occasion de l'envoi en Chine d'une mission catholique pour recueillir sur place les renseignements utiles. Ceux-ci, comme ceux concernant les autres branches de l'industrie de la soie, eurent une influence positive quant au développement de la sériciculture française. Dès la fin du xvIII siècle, la situation changea rapidement à la suite de la révolution industrielle en Angleterre ${ }^{2}$. La vulgarisation de l'industrie de la soie en Occident, due à une adoption de plus en plus ample de procédés mécaniques, accrut la demande en soie brute en même temps que l'exigence d'une certaine qualité. Dès le début des années 1840, on connut en Europe des changements considérables sur les plans politique, social et technique. Les relations entre I'Extrême-Orient et l'Extrême-Occident évoluèrent rapidement. La demande de l'industrie française concernant la sériciculture chinoise se modifia et les objectifs et les moyens d'investigation en Chine ne furent plus les mêmes. Toutefois, cette histoire fascinante de la circulation du savoir séricicole, notamment la partie concernant I'inspiration des savants français au XVIII ${ }^{e}$ siècle, pour le développement de leur industrie séricicole, est négligée par la plupart des historiens modernes qui ont, par ailleurs, fait de nombreuses études sur les échanges sino-européens grâce aux investigations des jésuites en Chine $^{3}$. II existe en revanche un grand nombre d'étu-

2. L'auteur a soutenu, en 2002, à I'EHESS, sa thèse, intitulée « L'industrie de la soie en France et en Chine de la fin du xviiie au début du xxe siècle : échanges technologiques, stylistiques et commerciaux. » Le présent article repose sur une partie de ce travail qui couvre la sériciculture, la confection de soieries et leur commerce ainsi que les échanges techniques concernant l'ensemble du travail de la soie.

3. L'influence chinoise sur l'Europe aux $x V \| l^{e}-x V I I l^{e}$ siècles couvre de nombreux domaines (philosophie, littéraire, architecture, mode et plusieurs secteurs de fabrication comme la porcelaine et le thé, etc.) ; elle conduisit à la mode des « chinoiseries ». L'influence européenne en Chine de même époque était en revanche plus des concernant l'introduction en Chine des techniques étrangères (européennes et japonaises) de la soie de la fin du XIX $x^{e}$ siècle au début du $x x^{e}$ siècle ${ }^{4}$.

Dans le présent article, nous nous intéresserons à la circulation du savoir séricicole entre la France et la Chine, véhiculée par les plus importantes enquêtes menées par les Français de la fin du xvII à la fin du XIX $X^{\text {e }}$ siècle, en tenant compte des objectifs respectifs de chaque prospection, des divers moyens adoptés par les enquêteurs ainsi que des résultats obtenus par ces expéditions. Inscrites dans une époque de grandes évolutions dans de multiples domaines (scientifiques, techniques, politiques et sociaux, etc.), ces enquêtes seront placées dans leur contexte historique, ce qui permet de mieux cerner les différentes natures propres à chacune. À cause d'une politique de "fermeture », ces enquêtes ont souvent été négligées par les Chinois. La présente étude est fondée essentiellement sur des documents français et anglais, mais aussi sur les ouvrages chinois de sériciculture rapportés en France.

restreinte et se limita à la cour et parmi certains lettrés dans le domaine des sciences (mathématiques, astronomie, etc.) et des arts (peinture, architecture, etc.) Le commerce maritime à Canton apporta aussi une certaine influence sur la peinture et quelques fabrications artisanales telles que la soieries et la porcelaine. ॥ existe une bibliographie importante sur le sujet, par exemple : Ronan C. E., Oh B. B.C. [dir.], East meets West: the Jesuits in China, 1582-1773: papers read at a symposium sponsored by Loyola University, Chicago, October 1982, Chicago, Loyola University Press, 1988, XXXIII-332 p. ; Lee Thomas H. C. (éd), China and Europe, Images and influences in sixteenth to eighteenth centuries, Hong Kong, The Chinese University Press, 1991, 356 p. ; Jami C., Delahaye H., L'Europe en Chine : interactions scientifiques, religieuses et culturelles aux XVII et XVIII siècles, Paris, Collège de France, Institut des Hautes Études Chinoises, 1993, 255 p. ; Pan Jixing, Zhongwai kexue zhi jiaoliu (Les échanges des sciences entre la Chine et l'étranger), Hong Kong, The University of Hong Kong, 1993, 578 p. ; Jacobson Dawn, Chinoiseries, London, Phaidon, 1993, $240 \mathrm{p}$.

4. Par exemple, Shannong Brown a fait une étude approfondie de I'histoire de cette filature. Voir « The Ewo Filature : a study in the transfer of technology in China in the nineteenth century ", dans Technology and Culture, juin 1979, vol. 20, n³, pp. 550568 ; Chen Tsu-yu, The Silk industry of modern China, 18601945, Taipei, Institute of Modern History Academia Sinica, 1989, 312 p. ; Li Pingsheng, "Lun wan Qing cansiye gailiang » (Essai sur l'amélioration de la sériciculture à la fin des Qing), Wenshizhe, 1994, n³, pp. 90-97 ; Bell L. S., One industry, two Chinas, Silk filatures and peasant-family production in Wuxi county, 18651937, Californie, Stanford University Press, 1999, 290 p. 


\section{Enquêtes des jésuites de l'extrême fin du XVII ${ }^{e}$ siècle au XVIII ${ }^{e}$ siècle}

En 1685, ayant décidé de patronner une mission catholique, Louis XIV envoya en Chine cinq jésuites « mathématiciens du Roi » qui, outre leur vocation de propager la foi chrétienne, avaient le double objectif d'effectuer des enquêtes sur les industries chinoises et d'établir des relations commerciales entre la France et la Chine. Avant leur départ, les jésuites reçurent du ministre Louvois ${ }^{5}$ un questionnaire sur les industries chinoises, dont celle de la soie.

Suivant le modèle de leurs prédécesseurs de la mission portugaise, les jésuites purent rester à la cour et jouir de la liberté de circuler à l'intérieur de la Chine, bénéficiant de l'intérêt particulier pour les sciences et les arts européens porté par les premiers empereurs Qing (1644-1911), Kangxi (1644-1722), Yongzheng (1723-1735) et Qianlong (1736-1795). À l'époque, il était strictement interdit aux étrangers de pénétrer à l'intérieur de l'Empire. Seules les ambassades envoyées par les royaumes tributaires pouvaient obtenir l'autorisation d'offrir leurs présents au Trône à Pékin. Les jésuites parvinrent à acquérir des ouvrages chinois, dont ceux de sériciculture, des graines de mûrier et des plantes industrielles ${ }^{6}$, grâce à des échanges avec les empereurs et les lettrés, mais aussi avec des jardiniers de la cour. Ils recueillirent aussi des renseignements utiles, soit auprès des artisans chinois (cultivateurs et jardiniers, etc.), soit par leurs propres observations. C'est ainsi que le $P$. d'Incarville (1706-1757) put rédiger son mémoire «Sur les vers à soie sauvages ${ }^{8}$, qui fut publié

5. Ce questionnaire fut remis au P. Couplet qui fut chargé de recruter des missionnaires en renfort.

6. Dumoulin-Genest Marie-Pierre, «L'introduction et l'acclimatation des plantes chinoises en France au xvIII siècle », Paris, thèse de doctorat non publiée de l'École des hautes études en sciences sociales, 1994, 3 vol. L'auteur décrit, avec beaucoup de soin, les moyens par lesquels les missionnaires réussirent à se procurer des graines ainsi que la manière dont elles furent transportées.

7. Pierre Noël Le Chéron d'Incarville, embarqué à Lorient sur le Jason le 19 janvier 1740, arriva à Canton le 15 janvier 1741. À Pékin, il fut botaniste et directeur des jardins impériaux et introduisit en France des plantes nouvelles. Voir Dehergne Joseph, Répertoire des jésuites de Chine, de 1552 à 1800, Roma, Institutum Historicum S. I., Paris, Letouzey \& Ané, 1973, pp. 128-129.

8. Voir la note de Matthieu Bonafous qui se trouve dans le manuscrit du P. d'Incarville, Bibliothèque municipale de Lyon, Ms. 6060 : Recueil de dessins chinois : vers à soie et travail de la soie (legs Bonafous). en 1777 par le P. Cibot (1727-1780)9 dans les Mémoires concernant les Chinois ${ }^{10}$, puis inséré dans la traduction de Stanislas Julien (1799-1873), intitulée Résumé des principaux traités chinois sur la culture des mûriers et l'éducation des vers à soie (1837). Les jésuites maintinrent aussi une correspondance étroite avec les académiciens de Paris, d'Europe et de Russie, auxquels ils envoyaient des renseignements et des échantillons demandés, et ce à l'aide des négociants de la Compagnie française des Indes.

Des résultats d'enquêtes menées par les jésuites en Chine furent publiés pour la première fois par le $P$. Le Comte dans ses Nouveaux mémoires ${ }^{11}$. Les Européens pouvaient y avoir une première impression de la fabrication chinoise des soies, des soieries et de leur consommation domestique. Mais c'est la Description de la Chine ${ }^{12}$, publiée en 1735 par le P. Du Halde (16741743), qui eut une influence profonde sur la sériciculture française. Se basant sur les informations recueillies par les jésuites en Chine, cet auteur y recueillit un extrait du Nongzheng quanshu (Traité complet d'agriculture) de Xu Guangqi (1562-1633) ${ }^{13}$, traduit par le P. Dentrecolles (1664-1741) ${ }^{14}$. Grâce à une large dif-

9. Embarqué le 7 mars 1758 à Lorient, Pierre-Martial Cibot, arriva à Pékin le 6 juin 1760. II était mécanicien, botaniste et polygraphe. Voir Dehergne J., op. cit., p. 176.

10. Mémoires concernant l'histoire, les sciences, les arts, les moeurs, les usages des chinois, Paris, Nyon, 1776-1789, t. II, pp. 575-601.

11. Le Comte Louis, Nouveaux mémoires sur l'état présent de la Chine, Paris, Jean Anisson, 1696-1698, 3 vol.

12. Du Halde Jean-Baptiste, Description géographique, historique, chronologique, politique, et physique de la Chine et de la Tartarie chinoise enrichie des cartes générales et particulières de ces pays, de la Carte générale et des Cartes particulières du Tibet et de la Corée, La Haye, chez Henri Scheurleer, 1735 (2 éd. en 1736), 4 vol.. Pour plus de détails sur la compilation de cet ouvrage voir : Landry-Deron Isabelle, La preuve par la Chine : la «Description» de J.-B. Du Halde, jésuite, 1735, Paris, Éd. de l'École des hautes études en sciences sociales, 2002, 428 p.

13. Baptisé en 1603 sous un nom chrétien, Paul, Xu Guangqi, natif de Shanghai, occupa le poste de grand ministre à la fin des Ming (1368-1644). Pendant les persécutions religieuses, il participa à la protection des jésuites. Son ouvrage intitulé Nongzheng quanshu fut publié après son décès par son fils en 1639, et importé en France, dans la première moitié du xvIII siècle par les Jésuites.

14. François-Xavier Dentrecolles, ou d'Entrecolles, débarqua le 21 février 1698 à « Emoi » [Amoy] et arriva à Pékin le 30 décembre 1719. Voir Dehergne J., op. cit., pp. 73-74. Selon Camille de Rochemonteix, Joseph Amiot, et les Derniers Survivants de la Mission Française à Pékin (1750-1795), Paris, Librairie Alphonse Picard et fils, 1915, p. XXXVII, il naquit le 5 février 1662. 
fusion de l'ouvrage du P. Du Halde, notamment par I'édition pirate éditée en 1736, des Français et même des Européens purent avoir une connaissance de techniques séricicoles chinoises.

Rappelons qu'au xvIII siècle, la sériciculture française subit de nombreuses maladies des vers à soie qui anéantirent souvent des magnaneries* entières. La soie produite était d'ailleurs d'une qualité grossière à cause des feuilles de mûrier données aux vers. Le savoir chinois publié dans La Description de la Chine offrit aux savants français de riches renseignements pratiques dont ils s'inspirèrent pour mener des expériences et pour mieux observer les bombyx. Mais au début de son introduction, l'influence chinoise se limita à certains domaines concrets et faciles à accepter, comme l'usage de filets (fig. 1) dans le délitement des vers à soie, l'élevage hâté, la construction de magnaneries et certaines méthodes de la culture du mûrier. Son influence allait s'intensifier en suivant les progrès des sciences et des techniques.

Au début des années 1760, selon le témoignage de L. Pomier ${ }^{15}$, « on se sert déjà fort avantageusement de ces filets dans plusieurs endroits de la Province ${ }^{16}$ ». Celui-ci recommanda vivement aux éleveurs français cette « méthode fort simple \& fort commode» des Chinois qu'il qualifia d' « excellente ». L'usage de filets avait pour avantage de pouvoir déplacer les vers avec plus d'efficacité et de sécurité ${ }^{17}$. Ils permettaient désormais de nettoyer plus fréquemment les litières* des vers à soie. Au cours de la seconde moitié $d u$ xvIII ${ }^{e}$ siècle, les savants furent d'avis de garder une couche épaisse de litière même par temps frais ${ }^{18}$; les éleveurs les accumulaient pour maintenir une chaleur élevée dans les claies. Ils ignoraient en revanche que la fermentation des ex-

15. Ingénieur des ponts-et-chaussées, L. Pomier a laissé des ouvrages sur la sériciculture : L'art de cultiver les mûriers blancs, d'élever des vers à soye et de tirer la soie des cocons, Paris, 1754 chez Vve Lottin et J. H. Butard, XXIV-256-6p ; Traité sur la culture des mûriers blancs, la manière d'élever les vers à soie, et l'usage $q u$ 'on doit faire des cocons, Orléans, impr. de Couret de Villeneuve, 1763, 288-8 p., suivi de Précis sur l'éducation des vers à soie, Tours, chez F. Lambert, impr. Du Roi \& de la Société R. d'Agric. De la Gén. De Tours, 1763.

16. Pomier L., Précis sur l'éducation des vers à soie, p. 54.

17. Avec les méthodes traditionnelles françaises, les vers risquaient de chuter pendant cette opération, ce qui entraînait des maladies par la suite.

18. Par exemple, Boissier de Sauvages, Art d'élever des vers à soie, Avignon, J.-J. Niel, 1788, p. 118. II conseilla d'enlever la litière " lorsqu'elle acquiert plus de deux doigts d'épaisseur, ou lorsqu'en passant la main par-dessous on sent qu'elle est humide ».
Fig.1. Filet pour délitement des vers à soie. Extrait de Stanislas Julien, op. cit. note 40.

créments et des brisures de feuilles pouvait produire un gaz nuisible aux vers et former dans le même temps un lit idéal pour multiplier les germes de maladies.

L'abbé Boissier de Sauvages (1710-1795) ${ }^{19}$, membre de plusieurs organisations savantes françaises et italiennes, est l'auteur du traité séricicole français du XVIII ${ }^{e}$ siècle qui prêta le plus

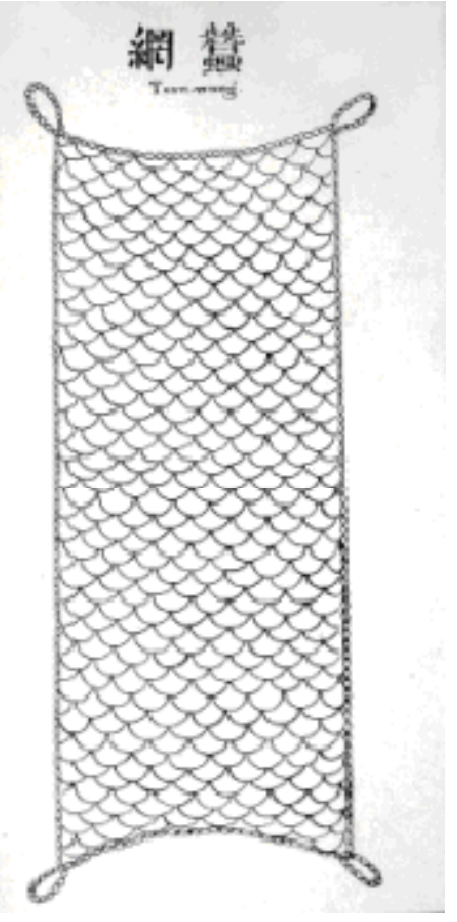
d'attention aux méthodes chinoises. Dans ses Mémoires sur l'éducation des vers à soie, il évoqua plusieurs méthodes chinoises et les compara avec celles de France et d'Italie. Initié par le savoir chinois, il entreprit des expériences $d^{\prime}$ " « élevage hâté », qui consistait à amener les vers à former leurs cocons dans un délai optimal en suivant certains critères, comme leur alimentation et les conditions dans les chambres d'élevage. En maintenant la magnanerie à une certaine température adaptée au développement des vers, la durée d'élevage fut réduite à vingt-quatre jours ${ }^{20}$, alors qu'elle était de quarante à plus de soixante jours dans une magnanerie traditionnelle. L'élevage hâté permettait de réduire la quantité de feuilles consommées tout en augmentant la productivité de la soie et en diminuant le risque de maladies

19. Pierre-Augustin de Boissier de (ou des) Sauvages, né à Alès en 1710 et mort dans la même ville en 1795. À 60 ans passés, il décida de se faire ordonner prêtre. II fit à deux reprises le voyage d'Italie et devint membre de la Société Royale des Sciences de Montpellier, de I'Académie de I'Institut de Bologne et de celle de Florence. II a laissé plusieurs ouvrages, notamment sur l'élevage des vers à soie: Mémoire sur les muscardins (1748); Mémoire sur l'éducation des vers à soie (1762), réédité sous le titre d'Art d'élever des vers à soie (1788) et une nouvelle édition par Luppi G., L'art d'élever les vers à soie par l'abbé Boissier des Sauvages, nouvelle édition tirée de l'édition de 1788, ordonnée et annotée à I'usage des sériciculteurs modernes, Lyon, Le Moniteur des soies, 1881, p. XVI.

20. En ce qui concerne les méthodes chinoises de sériciculture, voir Du Halde, op. cit., 1735, t. II, pp. 208-223 ; 1736, t. II, p. 251-267. 


\section{Enquêtes françaises sur la sériciculture chinoise et leur influence, fin $\mathrm{XVII}^{\mathrm{e}}$-fin $\mathrm{XIX}^{\mathrm{e}}$ siècles}

des vers. L'éleveur pouvait ainsi obtenir plus de profit et même réduire les frais de combustible. Pour faciliter la gestion des conditions de magnanerie (température, clarté et humidité...), il proposa une installation de magnanerie équipée d'un grand nombre d'ouvertures (portes, fenêtres et soupiraux...), ce qui en facilitait I'aération et permettait d'avoir un espace plus salubre que celui de la tradition.

Le Mémoires sur l'éducation des Vers à soie de Boissier de Sauvages a surtout été lu par des propriétaires de différentes provinces, qui déclarèrent avoir obtenu des succès en suivant ces instructions ${ }^{21}$. Dans l'impossibilité de faire suivre par des "magnaniers en titre » ces nouvelles méthodes, ces propriétaires formèrent eux-mêmes leurs employés néophytes dans l'élevage des vers à soie en s'appuyant sur cet ouvrage. En 1788, l'ouvrage fut réédité sous le titre L'Art d'élever des vers à soie et contribua au développement de la sériciculture dans les Cévennes ${ }^{22}$. À la fin du xvIII siècle, la sériciculture permit aux nombreux habitants cévenols de s'enrichir. Ils investirent ces gains dans la construction d'habitations plus saines, ce qui offrit de meilleures conditions à l'élevage des vers à soie ${ }^{23}$. Au début du XIX ${ }^{\mathrm{e}}$ siècle, s'inspirant du savoir chinois, un savant italien, Vincent Dandolo (1758-1819), ancien administrateur général de la Dalmatie et membre de I'Institut de Milan, conçut trois modèles de magnaneries de dimensions différentes, adaptées à la capacité des foyers d'éleveurs. La sériciculture cévenole put ainsi réaliser de remarquables progrès.

Les Français et les Italiens enviaient la soie chinoise, dite Sina ou Nankin ; ils cherchèrent à produire une soie aussi fine et d'une blancheur immaculée. Toutefois, dans l'industrie française traditionnelle de la soierie, seuls les fils de soie moulinés, décreusés et teints furent utilisés. Lorsque les soieries lourdes et de couleurs foncées furent à la mode, les soies de teinte naturelle n'offraient aucun inconvénient pour la confection. La soie Sina était exigée pour la fabrication de certains articles (par exemple des gazes, tulles, blondes et bas

21. Boissier de Sauvages, Art d'élever des vers à soie, pp. xiij-xiv. 22. Travier Daniel, « La soie dans la vie traditionnelle de la Cévenne », dans Actes du Ve Colloque surle patrimoine industriel, Alès, 19-21 octobre 1983, Paris, Comité d'information et de liaison pour l'archéologie, l'étude et la mise en valeur du patrimoine lindustriel (CILAC), 1984, pp. 12-24.

23. Jean-Paul Chabrol, Les Seigneurs de la soie, trois siècles de la vie d'une famille cévenole (XVie-xIx ), Presse du Languedoc, 1994, pp. 119-127. de soie, etc. ${ }^{24}$ ). À la fin du règne de Louis $\mathrm{XV}$, une mode d'étoffes légères et de couleurs claires, inspirée du style anglais, commença à conquérir l'Europe, ce qui coïncida avec la première réussite d'importation de graines chinoises de vers à soie à cocon blanc. En 1772, le missionnaire Jean Mathon de Fogrère, frère du procureur du roi au bailliage de Bourg-Argental, parvint à importer en France « une boîte de plomb, hermétiquement fermée, contenant quatre onces de [graines de] vers à soie $\operatorname{Sina}^{25} »$. Les années suivantes, on répéta à plusieurs reprises la même opération et on distribua ces graines aux éleveurs dans le Languedoc, les Cévennes, à Tours et dans d'autres régions. Mais I'acclimatation des vers Sina avait été difficile.

Au début des années 1780, le marché parisien fut envahi par de vaporeuses étoffes anglaises. Convaincus que le succès des Anglais était dû à la qualité supérieure de la soie chinoise, certains fabricants de Paris demandèrent au gouvernement d'encourager « la culture des belles soyes $d^{\prime}$ 'origine chinoise ${ }^{26} »$. Toutefois, la plupart des éleveurs français abandonnèrent rapidement cet élevage, parce qu'ils considéraient que les vers Sina étaient sujets aux maladies et exigeaient plus de soins ${ }^{27}$. L'élevage de ces vers ne leur apportait pas plus de bénéfice, parce que les manufacturiers français pouvaient s'approvisionner sur le marché londonien en soie Sina ou Nankin à un prix inférieur28.

Au début du siècle suivant, le Blocus continental (1808-1809) écarta le marché londonien des manufacturiers français. Le gouvernement français invita les cultivateurs à s'impliquer dans «l'éducation du ver à soie

24. Bulletin de la Société d'encouragement (ci-après, BSE), février 1815, n CXXVIII, pp. 37-38.

25. Voir Hedde Isidore, Description méthodique des produits divers recueillis dans un voyage en Chine, Saint-Étienne, Imprimerie de Théolier aîné, 1848, p. 85. Cependant, il faut attendre des recherches plus approfondies pour pouvoir conclure s'il s'agit de la première tentative pour introduire des graines chinoises en France ou bien de la première réussite d'importation de ces graines. Rappelons qu'à l'époque, les graines des vers à soie devaient subir des conditions peu confortables et la lenteur des voyages.

26. Archives de la Chambre de commerce et d'industrie de Paris, X- 4.00 (2), Soie et Gazes, " Manufactures de Paris », lettre adressée au Contrôleur général le 12 septembre 1784.

27. BSE, N LIX, mai 1809, p. 144 ; Archives du département de I'Hérault (ci-après, ADH), 6M 1600, « Le ministre de I'Intérieur, comte de l'Empire, à monsieur le Préfet du département de l'Hérault, Paris, le 31 mars $1809 »$.

28. $B S E, n^{\circ} \mathrm{CXXVIII,} \mathrm{février} 1815$, p. 38 
blanche ${ }^{29} »$, en leur garantissant un prix adéquat proposé par les fabricants lyonnais de gaze et de tulle. Dans le même temps, il demanda aux préfets de distribuer des traités, en espérant faire progresser les techniques séricicoles. La Société d'encouragement pour l'industrie nationale soutint cette campagne en proposant des prix « pour la culture des soies provenant de graines à cocon blanc $^{30}$ ». Elle fit connaître, dans son Bulletin de la Société d'encouragement, des techniques perfectionnées.

\section{Création de la «nouvelle école française » de sériciculture}

À la fin du xVIII siècle, à la suite d'événements politiques, religieux et financiers qui eurent lieu en France et en Chine, les jésuites disparurent peu à peu de la cour impériale de Chine. En vertu du traité de Paris (1763), la France perdit les Indes et fut écartée de l'Extrême-Orient. Jusqu'à l'envoi en Chine de la mission de Lagrené, à l'occasion de la signature du premier traité franco-chinois en 1844, les enquêtes françaises se réduisirent considérablement et furent menées par des hommes qui entraient secrètement à l'intérieur de l'Empire chinois.

En l'absence de contacts directs avec la Chine, certains érudits approfondirent leurs expériences sur la sériciculture en se basant sur les renseignements apportés antérieurement par des jésuites en Chine. Dans le même temps, on poursuivit des expéditions dans le monde entier tant pour collecter des méthodes utilisées par différentes ethnies que diverses sortes de mûriers et de vers à soie. Le but était de sélectionner les unes et les autres afin d'obtenir les meilleures par des moyens scientifiques nouvellement acquis. Plusieurs tentatives furent faites pour implanter la sériciculture dans les colonies françaises, notamment à Pondichéry, en Guyane et en Algérie.

Les années 1820-1830 furent marquées par l'essor de la sériciculture française : la surface des terres plantées de mûriers s'étendit31. En 1821, le botaniste G. Samuel Perrotet (1793-1870) importa des Philippines un mûrier chinois, le Morus multicaulis ${ }^{32}$. Facile à acclimater, le mûrier multicaule donna à la France, mais aussi à d'autres nations européennes (Allemagne,

29. $A D H, 7 M$, lettre du ministre de l'Intérieur, comte de l'Empire, à monsieur le Préfet du département de l'Hérault, Paris, le 31 mars 1809.

30. BSE, 1817, p. XXIV.

31. Annales de la Société séricicole (ci-après ASS), n 1, pp. 44-45.

32. Chinese Repository, 1846, vol. XV, p. 531.
Grande-Bretagne...) un espoir de grande extension de leur sériciculture, bien que certains savants craignissent sa résistance aux gelées ${ }^{33}$.

Dès la fin des années 1820, Camille Beauvais dirigea les bergeries de Sénart et y mena des expériences sur la plantation de mûriers et l'élevage des vers à soie. Suivant les méthodes chinoises, il s'attacha particulièrement à mettre au point I'élevage hâté et à réussir I'élevage de vers Sina. II cherchait surtout à établir des conditions optimales pour l'élevage des vers à soie à I'aide d'instruments scientifiques (tels que thermomètre, parcomètre...) et de moyens mécaniques, puisque les Chinois ne donnaient que des explications descriptives de leur pratique artisanale sans donner de chiffres précis. Dans le même temps, elle donnait des cours gratuits de sériciculture dans ses magnaneries-modèles.

Vers 1835, en collaboration avec d'Arcet (17771844) ${ }^{34}$, membre de I'Académie des sciences et de la Société royale et centrale d'agriculture, Beauvais installa dans sa grande magnanerie un système de « ventilation forcée ». Par le moyen d'un tarare et de cheminées, la magnanerie à la d'Arcet pouvait produire un « courant d'air symétrique et sans grande vitesse dans l'atelier ${ }^{35}$ ». ॥ permettait de purifier l'air de l'atelier dans un temps très bref. Cette installation gagna rapidement la plus grande estime des érudits français, qui en recommandèrent la vulgarisation $^{36}$. Le roi de France montra l'exemple en en construisant une à Villiers. La Société d'encouragement entreprit sa promulgation et publia dans son Bulletin des descriptions et des rapports concernant la « magnanerie salubre de d'Arcet ». Toutefois, cette installation était trop coûteuse pour les petits éleveurs et le système ne donna pas tout de suite des résultats satisfaisants. À côté de la grande magnanerie, Beauvais effectua des expé-

\section{ASS, $n^{\circ} 1,1837, \mathrm{pp} .158-160$}

34. Jean-Pierre-Joseph d'Arcet, ou Darcet.

35. D'Arcet, Considérations générales sur l'emploi de la ventilation forcée dans les magnaneries, Paris, Imprimerie Royale, 1837. pp. 19-20. Pour avoir plus de détails sur le système de Darcet, voir aussi BSE, 1835, p. 72.

36. Par exemple, dans sa lettre adressée à d'Arcet, le chimiste Payen considéra que la propagation du système d'Arcet était de " la plus haute importance » pour l'agriculture française, voir BSE, $n^{\circ}$ CCCLXXIII, juillet 1835, "Communication », pp. 368-369; dans son "Mémoire sur l'industrie de la production des soies », Henri Bourdon attribua les succès de Beauvais dans l'élevage des vers à soie à « sa vigilante expérience et à I'appareil ventilateur de M. d'Arcet », dans BSE, n CCCLXXXI, mars 1836, p. 99 et $\mathrm{H}$. De Villeneuve, Observation sur la ventilation des magnaneries, dans Eugène Robert, Conseils aux magnaniers de la nouvelle école séricicole, Marseille, Adolphe Barlatier, 1839. 
riences dans sa petite magnanerie et y développa un système permettant d'obtenir « des produits remarquables et une récolte très abondante avec une main-d'œuvre économique ${ }^{37} »$, système beaucoup plus apprécié des éleveurs français. Inspiré du savoir chinois, Beauvais devint le fondateur de "l'école séricicole française ${ }^{38}$ ».

Soutenues par le gouvernement, on vit alors apparaître partout en France des magnaneries modèles. Camille Beauvais fut souvent sollicité, comme d'autres savants intéressés par la sériciculture, et contribua à la vulgarisation de la sériciculture en France et dans ses colonies. Peltzer, un des disciples de Beauvais, fut envoyé dans le département du Vaucluse pour y diriger un élevage modèle. La Société d'agriculture et de commerce du Var envoya son secrétaire J.-F. Michel suivre les cours aux bergeries de Sénart. Son rapport, adressé au préfet de son département, fut publié en 1840, sous le titre de Résumé des leçons de $M$. Camille Beauvais ${ }^{39}$.

Satisfait des résultats obtenus en suivant les méthodes chinoises, le directeur des bergeries de Sénart suggéra au ministre de l'Intérieur de faire traduire les traités de sériciculture conservés en France ${ }^{40}$. Stanislas Julien, professeur au Collège de France, fut chargé de leur traduction et publia en 1837 son Résumé des principaux traités chinois sur la culture des mûriers et l'éducation des vers à soie. Pour ce travail, il entretint une correspondance avec Matthieu Bonafous (17931852), directeur du Jardin royal d'agriculture de Turin. La même année, sur l'initiative de Beauvais, fut créée à Paris la Société séricicole, au sein de laquelle les membres faisaient part des résultats de leurs expériences et discutaient de l'intérêt des dernières techniques. Ayant des contacts étroits avec des académies et d'autres sociétés savantes, la Société devint un lieu d'échange actif; elle publiait dans l'Annale de la Société séricicole des nouvelles techniques de sériciculture, de tirage de la soie, et la situation de l'industrie de la soie.

La propagation de la sériciculture jouissait d'un solide soutien du gouvernement français parce qu'elle était considérée comme une activité « de la plus haute

37. BSE, $n^{\circ}$ CCCLXXIII, juillet 1835, "Communication», p. 368. 38. Eugène Robert, op. cit., p. 25.

39. J.-F. Michel, Résumé des leçons de M. Camille Beauvais, sur la construction des magnaneries à la d'Arcet, l'éducation des vers à soie, la culture et la taille des mûriers, rapport adressé à M. Lemarchand de la Faverie (Préfet du Département du Var), Brignoles, imprimerie De Perreymond-Dufort, 1840, p. 39.

40. Julien Stanislas, Résumé des principaux traités chinois sur la culture des mûriers et l'éducation des vers à soie, Paris, Imprimerie Royale, 1837, 224 p. + 10 pl., préface.

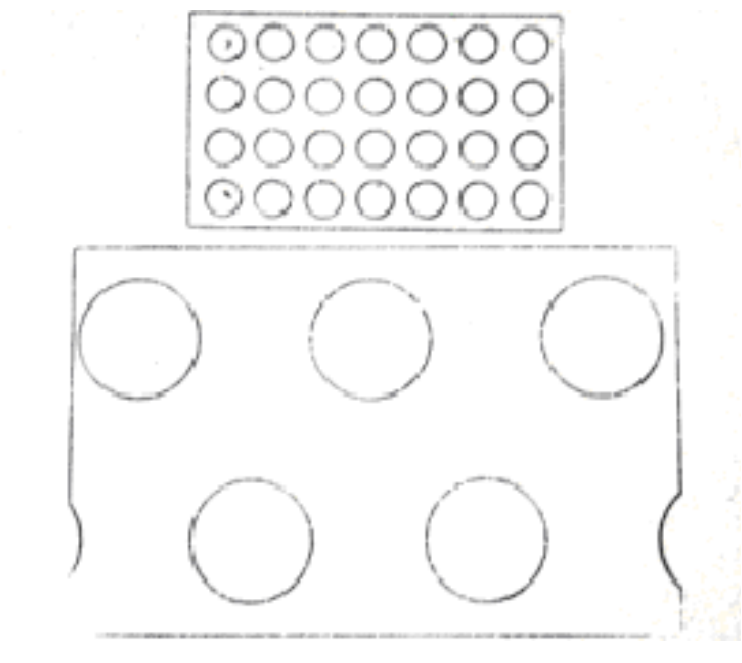

Fig. 2. Papier sans fin perforé, d'après E. Maillot, dans Maillot E., Lambert F., Traité sur le ver à soie du mûrier et sur le mûrier, Montpellier, Coulet et fils, éd., Paris, Masson et Cie, 1905.

importance » pour l'agriculture, permettant à la France de s'affranchir du " tribut » d'importation de soies étrangères ${ }^{41}$. Mais le problème de maladies des vers à soie demeurait : dès le début des années 1840, les récoltes de cocons devinrent irrégulières. En 1847, Félix-Édouard Guérin-Méneville (1799-1874), zoologiste et membre de la Société royale et centrale d'agriculture de Paris, fut chargé par le ministre de l'Agriculture et du Commerce d'en faire des études. Il entra aussitôt en relation avec Eugène Robert et mena des expériences dans sa magnanerie-modèle de Saint-Tulle, associant ainsi véritablement pratique et théorie. Eugène Robert avait modifié les procédés de délitage des vers conçus par Beauvais en remplaçant les filets par du papier sans fin perforé (fig. 2).

\section{La délégation commerciale attachée à la mission en Chine de Lagrené (1843-1846) ${ }^{42}$}

Avec la signature du traité de Nankin, en 1842, cinq ports chinois (Canton, Amoy [Xiamen], Fuzhou, Ningbo

41. BSE, $n^{\circ}$ CCCLXXIII, juillet 1835, "Communication», p. 368. 42. Plusieurs communications présentées au colloque intitulé Les échanges franco-chinois du xix siècle : la mission en Chine de Lagrené (1843-1846), les 3 et 4 juin 2004 au Collège de France à Paris, avec l'aide du Centre d'études sur la Chine moderne et contemporaine (CECMC), EHESS, et de la Maison des sciences de I'homme, abordent les échanges entre la France et la Chine dans plusieurs domaines (textiles, peintures, construction navale, etc.). 
et Shanghai) furent ouverts au commerce avec I'Occident. À l'instar de l'Angleterre, la France envoya sa première ambassade, dirigée par Théodose de Lagrené (1800-1862), ministre plénipotentiaire de France en Chine, pour traiter avec l'Empire et obtenir les mêmes avantages et garanties que les Anglais.

Sur la demande insistante des chambres de commerce qui souhaitaient « voir s'ouvrir à leurs produits les nouveaux et importants marchés de l'Indo-Chine ${ }^{43}$ », une délégation commerciale fut attachée à cette ambassade. Quatre délégués, dont Isidore Hedde (18011880) représentant l'industrie des soies et des soieries, et Natalis Rondot (1821-1900), l'industrie des laines et des vins de Champagne, furent chargés de mener des enquêtes détaillées sur les marchés et industries de Chine et d'Asie du Sud-Est, dans le dessein d'informer les manufacturiers français qui voulaient développer leur commerce, des goûts et des conditions des marchés de ces pays éloignés. Dans le même temps, il leur fut demandé de repérer les produits locaux pouvant intéresser le marché français, ce qui permettrait de charger les navires à leur retour.

Dès son arrivée à Canton le 18 octobre 1844, Isidore Hedde entra en relation avec de nombreuses personnes (fonctionnaires, marchands, agents diplomatiques, missionnaires, jardiniers...), indigènes, françaises ou occidentales, qui l'aidèrent beaucoup pour remplir sa mission ${ }^{44}$. Le 25 novembre 1844, accompagné de Jules Itier (1802-1877), délégué des douanes attaché à la mission de Lagrené, Isidore Hedde fit une expédition sur une voie fluviale intérieure reliant Canton et Macao ${ }^{45}$. Pendant cette expédition, il recueillit beaucoup d'informations sur la sériciculture cantonaise et observa la région séricicole alentour. II fit aussi, auprès d'ateliers de peinture situés dans les factoreries de Canton (en particulier ceux de Tingqua, de Sunqua et de Yeouqua $\left.{ }^{46}\right)$, des commandes de dessins représentant

43. Archives nationales (ci-après, AN), 12F/2589, « Correspondance relative à la Mission en Chine, du 20 avril 1843 au 23 février 1844 », Lettre de S. E. le ministre de l'Agriculture et du Commerce à $S$. E. le ministre des Affaires Etrangères du 14 septembre 1843.

44. Pour plus de détails sur les enquêtes menées par Hedde, voir Mau Chuan-Hui, "Isidore Hedde et la soie chinoise », dans La revue du Musée des Arts et Métiers, mai 2004, n 41, pp. 32-42.

45. Isidore Hedde a publié lé récit de ce voyage dans le Chinese Repository, 1848, vol. XVII, pp. 423-431.

46. Peintres connus de l'époque pour les peintures d'exportation, particulièrement vers l'Europe. II existe aussi des albums représentant d'autres industries textiles : thé, coton et d'autres fibres textiles, etc. en détail le travail de la soie, de la plantation du mûrier jusqu'à la confection des soieries et à leur commercialisation ${ }^{47}$. Aidé par des missionnaires protestants, Hedde parvint à visiter la sériciculture de Zhangzhou dans le Fujian $^{48}$. Après son retour en France, Isidore Hedde entretint des correspondances avec certains missionnaires en Chine. Dans ses lettres, il leur posait des questions sur les conditions de la sériciculture à l'intérieur de I'Empire, par exemple dans la province du Yunnan ${ }^{49}$.

Toutefois, les techniques de la sériciculture à Canton n'étaient pas des plus avancées. Isidore Hedde remarqua rapidement que les Cantonais ne pratiquaient que la plantation du mûrier par semis et par bouturage mais qu'ils ignoraient les techniques de greffe, permettant de modifier la qualité du mûrier. Ce sont les vers polyvoltins (vers ayant plusieurs générations au cours d'une année) élevés dans ces régions, et le mûrier multicaulis qui retinrent spécialement l'attention de notre délégué. II manifesta en revanche une admiration particulière pour la sériciculture du Jiangnan, où se trouvaient les plus importantes régions séricicoles de l'Empire. II conseilla d' « examiner les progrès des procédés modernes, l'emploi de la poudre des feuilles de mûriers par temps de disette, les étagères mobiles, les coconnières ingénieuses, les méthodes simples pour éviter le duvetage, enfin, tous les perfectionnements qui assurent aux produits du Che-Kiang [Zhejiang] et du Kiang-sou [Jiangsu] la supériorité sur ceux des autres provinces ${ }^{50} »$. Une grande quantité de graines de mûrier et de vers à soie venant de diverses régions fut adressée, pendant son voyage et après son retour en France, à la Société séricicole de Paris, et à d'autres sociétés savantes et jardins des plantes qui les distribuèrent par la suite à leurs membres pour des essais ${ }^{51}$. La Société séricicole lui avait demandé en particulier des graines de vers à soie d'origine chinoise en raison de la qualité de sa soie, supérieure à celle du Bengale.

47. Ces albums - à présent conservés dans différents établissements en France (Cabinet des estampes de la Bibliothèque nationale et Bibliothèque centrale du Conservatoire national des arts et métiers, etc.) - étaient des documents importants pour les Français de l'époque intéressés par les techniques chinoises. 48. Chinese Repository, 1847, vol. XVI, pp. 75-84

49. AN, F/12/2589.

50. Faits commerciaux, $n^{\circ} 12$, p. 95.

51. ASS, vol. 8, 1844, "séance du 7 février 1845 », p. 391 ; Hedde Isidore, Description méthodique des produits divers recueillis dans un voyage en Chine, Saint-Étienne, Imprimerie de Théolier aîné, 1848, pp. 106, 121 et 126-128. 
Après le retour de la délégation, des échantillons de soie furent soumis à des analyses et des essais industriels et les résultats furent rendus publics à l'occasion des expositions organisées à Paris (1846), à Lyon (1847) et à Saint-Étienne (1848). Un grand nombre de spécimens concernant les différentes espèces de mûriers et de vers à soie y furent exposés. On y trouva aussi des ustensiles employés pour la culture du mûrier et pour l'élevage des vers à soie. Ces expositions avaient comme premier objectif de faire connaître aux manufacturiers et fabricants français les produits venant de l'Orient.

Les enquêtes d'Isidore Hedde eurent une influence plus visible sur l'industrie française des soies et des soieries que sur la sériciculture ${ }^{52}$. Une très riche gamme de soies qu'il avait importées en France permettait aux industriels français, notamment ceux du moulinage, de faire des essais afin de mieux les adapter à leur fabrication, ce qui était utile pour exploiter le marché potentiel chinois de la soie. Dans les années 1850, lorsque les maladies des vers à soie anéantirent la sériciculture française, puis européenne, les industriels français se tournèrent vers le marché chinois. Ce commerce fort lucratif était alors monopolisé par des grandes maisons anglaises (Jardine, Matheson \& Co. et Dent, Beale \& Co.) et américaine (Russell \& Co.). Grâce aux efforts de Charles de Montigny (1805-1868), ancien chancelier de Lagrené lors de sa mission en Chine et premier agent consulaire français à Shanghai, en 1852, une maison française parvint à envoyer 85 balles de soie chinoise aux soyeux lyonnais directement de ce port nouvellement ouvert ${ }^{53}$. Mais il fallut attendre la fin des années 1860 pour voir le marché se déplacer de Londres à Lyon, ce qui coïncida avec le déclin de l'industrie de la soie et l'essor de la sidérurgie en Angleterre.

\section{Investigations faites sur I'initiative de Natalis Rondot}

À la suite de la seconde guerre de l'Opium, le traité de Tianjin, en 1858, ouvrit plus de ports chinois au commerce avec l'Occident et offrit à celui-ci plus d'avantages, notamment dans la fixation des tarifs douaniers

52. Pour plus de détails, voir note 1.

53. Rondot N., Commerce de la France avec la Chine, délibération prise sur le rapport de $\mathrm{M}$. Rondot, séance du 12 janvier 1860, Lyon, Imprimerie de Louis Perrin, 1860, p. 8 ; Maybon C. B., Fredet Jean, Histoire de la concession française de Changhai, 1929, p. 169. à l'intérieur de la Chine concernant les marchandises destinées à l'exportation ${ }^{54}$. Profitant de la possibilité de construire des établissements sur le territoire chinois, accordée conformément au même traité, les Anglais et les Américains se hâtèrent de créer des industries dans leurs concessions. La maison anglaise Jardine, Matheson et Co. se lança dans l'établissement de la première filature à Shanghai ${ }^{55}$. Malgré son succès sur le marché européen, à la fin des années 1860, la filature ferma ses portes à cause d'un déséquilibre de son bilan.

En 1867, Louis Pasteur parvint à identifier l'origine des maladies fatales des vers à soie, la pébrine et la flacherie. Il découvrit la méthode du grainage cellulaire* qui permettait d'obtenir des graines saines et d'arrêter ce fléau de l'histoire de la soie. Dès le début des années 1870, le prix des soies avait beaucoup baissé, à cause de la conjoncture de plusieurs facteurs : raccourcissement du voyage entre la Chine et l'Europe à la suite de la percée du canal de Suez et l'adoption de l'étalonor par les pays occidentaux. Grâce à la découverte de Pasteur, le retour de la soie italienne sur le marché international, auquel s'ajoutèrent de nouveaux marchés, Japon et Inde, vint renforcer cette tendance. En 1885, sur le marché lyonnais, les prix des soies connurent une baisse de $50 \%$ à $60 \%$ par rapport aux prix de $1868^{56}$. Les cultivateurs français ne s'intéressèrent plus à la sériciculture, malgré les subventions proposées par leur gouvernement. Les soies chinoises constituant le principal marché pour l'approvisionnement en matières

54. Conformément au traité de Tianjin, les marchandises occidentales importées en Chine et les produits chinois exportés pouvaient payer un droit de $2,5 \%$, à la douane maritime de leur lieu de débarquement pour les marchandises occidentales ou à la première douane rencontrée sur leur itinéraire vers la douane maritime pour les produits chinois. Ce droit était destiné par les Occidentaux à remplacer les taxes de transit requises à l'intérieur de chaque province de l'empire. Mais si le paiement des taxes de transit était plus avantageux, les marchands occidentaux pouvaient aussi choisir de payer celui-ci. En conséquence, les Occidentaux ne payaient qu'un droit de 7,5\% ad valorem au maximum de taxes pour les exportations des marchandises chinoises, notamment des matières premières, et pour les importations en Chine des produits occidentaux, en particulier manufacturés. Ceci mit les marchands et fabricants chinois dans une situation peu favorable face à la concurrence des produits occidentaux, puisqu'ils étaient chargés de taxes de plus en plus lourdes.

55. Shannong Brown a fait une étude approfondie de I'histoire de cette filature. Voir « The Ewo Filature : a study in the transfer of technology in China in the nineteenth century», in Technology and Culture, juin 1979, vol. 20, n³, pp. 550-568.

56. Chiffre obtenu selon la statistique donnée dans Natalis Rondot, La Soie, Paris, Imprimerie nationale, 1887, p. 4. 
premières de la France, les industriels français attachèrent par la suite de l'importance au développement de la sériciculture chinoise.

À la fin des années 1870, la diminution continuelle des récoltes de cocons en Chine, malgré l'augmentation de la surface des plantations de mûriers, inquiétait les Occidentaux. Natalis Rondot, alors représentant à Paris de la Chambre de commerce de Lyon, adressa en 1878 et en 1879 deux lettres à Robert Hart (18351911), inspecteur général des douanes maritimes à Pékin, pour solliciter de sa part une inspection générale de l'industrie chinoise de la soie. Dans sa deuxième lettre, Rondot joignit un questionnaire. Dans leurs rapports, les commissaires des douanes tenaient compte non seulement de la sériciculture des vers domestiques, mais encore de celle des vers à soie sauvages ${ }^{57}$. Certains rapports étaient accompagnés d'illustrations permettant de mieux comprendre les techniques de sériciculture et d'élevage des vers à soie (fig. 3). Si Rondot prêta particulièrement attention à l'état actuel de la sériciculture chinoise, c'est peut-être parce qu'en 1858 il assista à une commission de la Société royale impériale zoologique d'acclimatation pour la lecture du projet de I'expédition en Chine proposée par deux sériciculteurs scientifiques italiens, Castellani et Freschis8. À I'aide du consul de Shanghai, ceux-ci parvinrent à s'installer en 1859 aux environs de Huzhou, haut lieu de la sériciculture chinoise, et y effectuèrent des expériences qui avaient pour objectif de stopper le fléau des maladies des vers à soie. Des graines infectées des maladies fatales étaient emballées dans leurs bagages pour la réalisation du projet, malgré l'avertissement de l'agronome italien Cosimo Ridolfi (1794-1865) ${ }^{59}$.

57. Les résultats de cette prospection, entreprise en 1880, furent publiés en 1881 dans un numéro spécial de la collection des douanes maritimes: China, Imperial maritime customs, II. Special series : $n^{\circ} 3$, Silk, Shanghai : Statistical Departement of the Inspectorate General, 1881, 163 p.

58. Guérin-Méneville Félix-Édouard, «Rapport sur le projet de voyage en Chine de MM. Les comtes Castellani et Freschi ayant pour objectif d'étudier les vers à soie dans ce pays et d'y faire faire de la graine pour essayer de régénérer nos races atteintes depuis quelques années par l'épidémie de la Grattine », société impériale zoologique d'acclimatation, extrait du Bulletin de la Société impériale zoologique d'Acclimatation, $n^{\circ}$ de novembre 1858, p. 1.

59. Zanier Claudio, Alla ricerca del seme perduto, Sulla via della seta tra scienza e speculazione (1858-1862), Milano, Franco Angeli, 1993, p. 110.

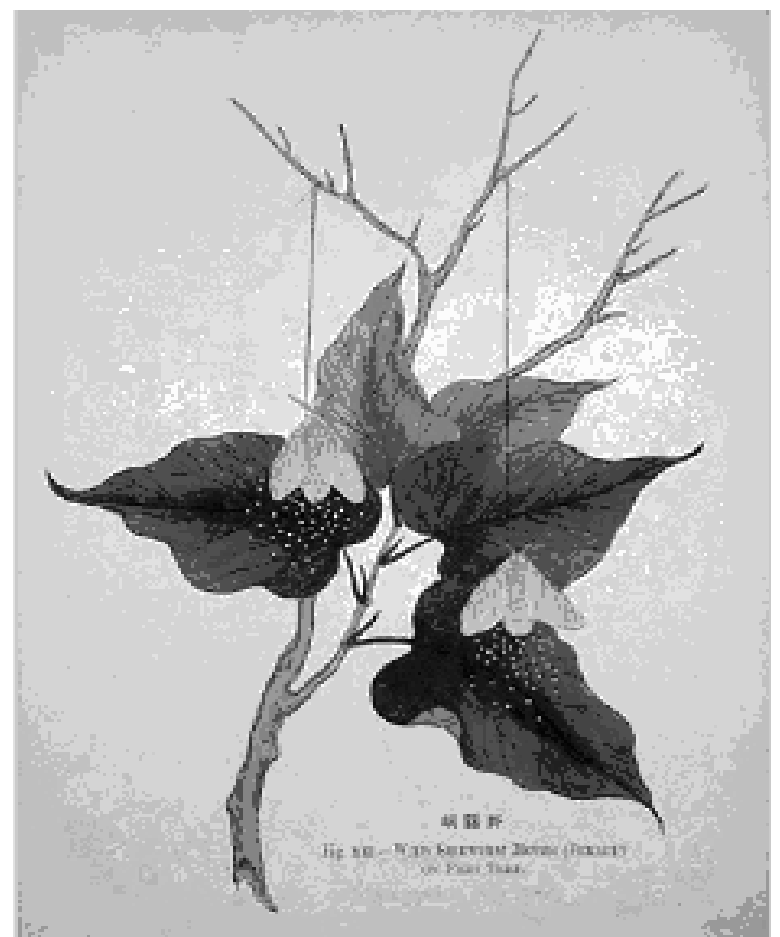

Fig. 3. Papillons des vers à soie sauvages attachés sur l'arbre pour le grainage. Extrait de Silk, bibliothèque de I'Institut des hautes études chinoises.

Après la fin de son contrat au Japon en 1875 pour la fondation de la filature de Tomioka ${ }^{60}$, Paul Brunat (1840-1903) accepta l'invitation de la maison américaine Russel \& Co., qui voulait suivre le modèle de Jardine, Matheson \& Co. pour créer à Shanghai une filature. Profitant de son séjour à Shanghai, il observa de près les élevages des vers à soie de la région ${ }^{61}$ et remarqua I'extension sérieuse de la pébrine dans la sériciculture chinoise. En 1883, il profita de son rendez-vous avec Li Hongzhang (1823-1901), gouverneur général du Zhili (équivalent du Hubei actuel) et ministre du Commerce de la mer du Nord, pour lui adresser un mémoire dans lequel il expliqua la situation inquiétante de la sériciculture chinoise, mais cela ne provoqua pas le moindre souci chez ce grand mandarin chinois ${ }^{62}$.

À la suite de l'enquête sur l'industrie chinoise de la soie faite sur sa demande, Natalis Rondot entretint

60. Shito Akira, Shinkoku sanshigyô ippan (L'état général de I'industrie de la soie des Qing), Tokyo, Seishi kensa sho, 1911, p. 73.

61. Bulletin des soies et des soieries (ci-après BSS), 20 septembre 1884, n³90, pp. 4-5.

62. Le rapport, complété par des notes écrites en septembre de la même année, fut publié dans le BSS du 6 octobre 1883 et des 26 janvier, 2 et 9 février 1884. 


\section{Enquêtes françaises sur la sériciculture chinoise et leur influence, fin XVII ${ }^{e}$-fin $\mathrm{XIX}^{\mathrm{e}}$ siècles}

une correspondance avec l'Allemand F. Kleinwächter ${ }^{63}$, commissaire des douanes à Zhenjiang, ville située dans une importante région séricicole. En 1886, après son retour d'Europe, Kleinwächter, affecté cette fois-ci comme commissaire de la douane de Ningbo, procéda à une enquête sur les maladies des vers à soie dans la sériciculture du Zhejiang en suivant simultanément deux démarches : d'une part, il envoya un questionnaire ${ }^{64}$ aux éleveurs habitant dans les régions séricicoles de la province en leur demandant de le lui retourner rempli et accompagné d'échantillons de graines, de cocons et de soie. D'autre part, il embaucha des sériciculteurs locaux pour faire des élevages expérimentaux dans la résidence de la douane maritime, et ce avec les graines achetées sur le marché. Par la suite, il envoya en France, pour les soumettre à une série d'examens, les échantillons que les sériciculteurs lui avaient envoyés en y joignant ceux issus de ses propres élevages expérimentaux. Le résultat de ces analyses confirma la sévérité de la contamination par la pébrine et la flacherie.

Sur le conseil de Natalis Rondot, Kleinwächter adressa des mémoires au bureau des Affaires étrangères pour demander à celui-ci de prendre des mesures nécessaires pour stopper ce fléau ${ }^{65}$. Face à l'indifférence du bureau, il décida d'envoyer à Montpellier son éleveur chinois, qui s'occupait de l'élevage dans la résidence de la douane, accompagné d'un interprète chinois, pour étudier les techniques modernes de la sériciculture. Leur apprentissage était loin d'être satisfaisant à cause d'une absence totale de connaissances en sciences ; il se limita à l'utilisation du microscope afin de pouvoir produire des graines saines. Même après le retour en Chine de ces apprentis, aucun fonctionnaire

63. Entré en 1865 dans la douane maritime chinoise, il fut nommé, l'année suivante, commissaire de la douane de Zhenjiang. Dès lors, il occupa le poste de commissaire à Ningbo, Shantou et Guangdong jusqu'en 1889, année où il démissionna. Pendant son occupation du poste de douane, il s'adonna à l'amélioration de la sériciculture chinoise et rédigea plusieurs textes pour inciter des autorités centrales des Qing à adopter certaines techniques de sériciculture.

64. Je n'ai pas encore pu me procurer ces brochures. Mais, d'après le texte de Kleinwächter, shu signifie un ouvrage ou une brochure. Suivant sa description, il devait s'agir de formulaires d'enquête comparables aux journaux que tiennent les sériciculteurs chinois sur leurs élevages décrits étape par étape. Voir Kleinwächter F., Canwu tiaochen (Exposé méthodique de sériciculture) [s.I.n.d.], p. $2 a$.

65. Kleinwächter F., idem. et son « Canwu tushuo » (description et illustrations de sériciculture), dans Xizheng congshu (Collection concernant les affaires occidentales), 1889. de l'Empire ne montra le moindre intérêt pour le projet de Kleinwächter. II fallut attendre que le préfet de Hangzhou, Lin Qi (1839-1900)66 ${ }^{6}$ s'inspire de ce projet pour créer en 1897 dans sa préfecture la première école de sériciculture en Chine ${ }^{67}$. Cela s'inscrivait dans le cadre historique complexe et instable que connut la Chine à la fin du xix siècle. Après la signature du traité de Shimonoseki en 1895, les lettrés réformistes se mobilisèrent avec énergie pour sauver I'Empire en introduisant les sciences et les techniques modernes de l'étranger. On vit apparaître les unes après les autres des sociétés savantes et des organisations scientifiques. Pendant les premiers mois de l'ouverture de l'école, l'éleveur de Kleinwätcher fut chargé de l'enseignement des techniques du grainage. II démissionna rapidement et laissa cet enseignement à des professeurs japonais. Ce fut d'ailleurs par l'intermédiaire du Japon que des instruments scientifiques, comme le microscope et le thermomètre, et des techniques modernes de sériciculture furent introduits en Chine. Mais le coût élevé des équipements et les connaissances scientifiques exigées concernant la sériciculture moderne entravèrent sa vulgarisation et la confinèrent derrière les murs des établissements savants. II fallut attendre les années 1920 pour que les techniques modernes de la sériciculture puissent pénétrer dans les foyers de paysans éleveurs.

\section{Conclusion}

À travers ces enquêtes françaises qui s'étalent sur plus de deux siècles, nous observons un mouvement de vaet-vient dans l'introduction des techniques séricicoles. Les savants français des $X V I_{I I}{ }^{-}-X I X^{e}$ siècles ne se contentaient pas d'imiter aveuglement les procédés chinois de sériciculture. Ils cherchaient à les comprendre et à les expliquer en s'appuyant sur les sciences qui étaient en voie de développement. La sériciculture quitta peu à peu le domaine purement artisanal pour entrer dans un domaine scientifique. Les expériences empiriques des Chinois nourrirent les progrès des sciences et, à leur tour, celles-ci vinrent soutenir le développement de la

66. Zhu Xinyu, Qiu Liangru, "Canxue guan-Zhongguo diyi suo fangzhi xuexiao » (La première école de textile chinois), dans Zhongguo fangzhi shi ziliao, t. IV, mars 1981, pp. 37-43.

67. Pour plus d'informations sur cette histoire, voir Mau ChuanHui, "L'introduction en Chine des sciences et des techniques européennes concernant l'industrie de la soie après la guerre de I'Opium », dans Études chinoises, vol. XX, n 1-2 (printempsautomne 2002), pp. 201-237. 
sériciculture. Nous remarquons une influence chinoise grandissante en suivant l'avancement des recherches sur la sériciculture. À la fin du XIx $x^{\mathrm{e}}$ siècle, les puissances européennes imposèrent à la Chine, pays exportateur de techniques jusqu'à cette date, leurs techniques nouvellement mises au point, et ce, notamment, à cause d'une lourde pression économique pesant sur celle-ci. Dans cette histoire de la circulation du savoir séricicole de l'époque étudiée, dès le début, la Chine a manifesté une attitude passive. On se demande si cette indifférence ne repose pas sur le fait que ce pays a monopolisé durant très longtemps la maîtrise absolue de ces techniques. La France, quant à elle, pays importateur de soies et de techniques séricicoles étrangères, devint, dès la fin des années 1860, un pays exportateur de techniques séricicoles, notamment grâce à la méthode de grainage créée par Pasteur pour enrayer le désastre des maladies des vers à soie.

La balance de la direction des échanges techniques suivait l'évolution de l'appréciation française du savoir chinois. Avec les remarquables progrès des sciences et l'évolution sociale, les savants français du xIx siècle tentèrent de développer la sériciculture comme une industrie très productive. Malgré son aspect rudimentaire à cause des ustensiles utilisés dans l'élevage des vers à soie, le savoir chinois alimenta continuellement les recherches scientifiques menées en France, et ce jusqu'à la fin du xIXe siècle.

Étant une industrie lucrative, l'industrie séricicole française obtint continuellement le soutien des gouvernements de France. Avant l'ouverture de la Chine par différents traités signés après la première guerre de l'Opium, les jésuites en Chine furent les principaux enquêteurs sur les industries chinoises. Grâce aux négociants de la Compagnie française des Indes, les renseignements et documents recueillis purent être importés en France. Les Académies des sciences et les sociétés savantes provinciales furent les centres d'études et d'échanges permettant de faire progresser le savoir. Les notables (aristocrates, bourgeois et propriétaires fonciers) contribuèrent à la vulgarisation du savoir. La situation changea après 1842. Entreprise à l'initiative des gouvernements français ou des chambres de commerce, la réalisation des investigations a été faite grâce à l'intervention de gens de différents milieux (agents diplomatiques, militaires, missionnaires, industriels, marchands, etc.), et de diverses nationalités, occidentales et chinoises. La superposition des réseaux scientifiques, commerciaux et administratifs et leurs interrelations ont tissé une histoire riche et fascinante.

\section{Lexique}

Cabane : installation, constituée de rameaux de bruyère ou de tiges de paille, destinée à recevoir des vers ayant atteint leur maturité, leur servant de support pour former leur cocon.

Croisure : croisement de fils de soie sortis de filières* soit entre deux fils (croisure à la chambonne, voir fig. 4 ci-dessous), soit sur eux-mêmes pour former un autocroisement. L'existence d'une croisure permet aux brins de mieux adhérer entre eux - et ainsi de former un fil de soie aussi rond que possible - et d'éliminer I'humidité contenue dans le fil.

Façonné : tissu décoré de motifs obtenus pendant le tissage par le croisement des fils de chaîne et de trame.

Filière : passage se trouvant à la sortie de la bassine, lors de l'opération du tirage de la soie* , permettant de réunir un certain nombre de brins de cocons pour former le fil de soie (voir fig. 4 ci-dessous).

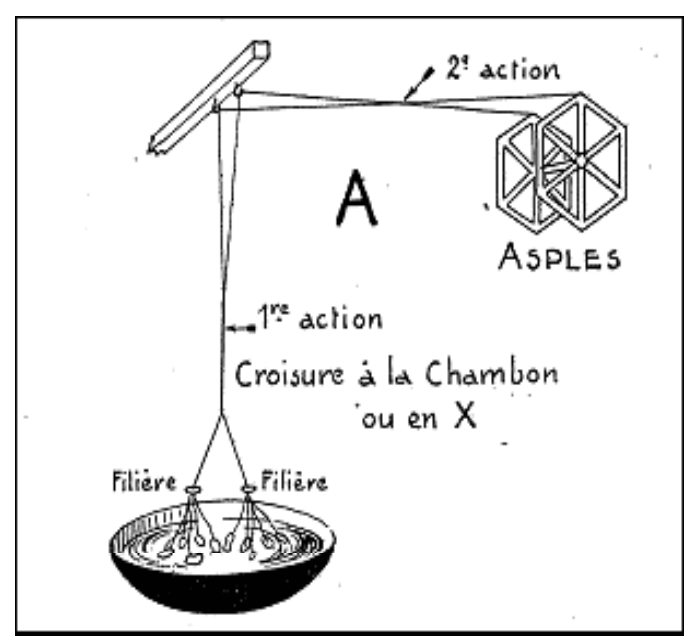

Flotte : écheveau de soie.

Grainage : suite d'opérations ayant pour objectif d'obtenir des graines* pour le prochain élevage. Des cocons mâles et femelles en nombre égal sont mis à part parmi les mieux formés et réservés pour ne pas subir le tirage* ; après l'accouplement des papillons, on les place sur une toile ou un papier spécial, afin que la femelle y ponde ses œufs.

Grainage cellulaire : système de grainage* conçu par Pasteur, permettant d'obtenir des graines saines, à la suite de sa découverte en 1867 du caractère héréditaire et contagieux de la pébrine. Il consiste à isoler les couples, ou au moins les papillons femelles et, à l'aide d'examens au microscope, à éliminer rigoureusement les œufs pondus par des papillons infectés. 
Graine [de ver à soie] : œufs des vers à soie.

Guindre ou asple : support rotatif qui sert à recevoir l'écheveau.

Litière des vers à soie : mélange d'excréments des vers à soie et du restant de feuilles de mûrier.

Magnanerie : local conçu spécialement pour l'élevage des vers à soie. De tels établissements n'existaient pas en Chine avant la fin du XIX siècle. Jusqu'à cette date, à l'arrivée de la saison d'élevage, les paysans éleveurs libéraient et aménageaient des pièces à l'intérieur même de leur habitation.

Montée : opération consistant à disposer les vers ayant atteint leur maturité sur une cabane* , ou bruyère, pour qu'ils y tissent leur cocon.

Moulinage (ou ouvraison) : ensemble d'opérations de torsion et de doublage de la soie grège pour lui don-ner plus de résistance afin qu'elle puisse résister à diverses manipulations pendant le tissage. On appelle la soie ainsi traitée soie moulinée, ou soie ouvrée. Suivant leur qualité et l'usage voulu, ces soies subissent différents procédés de torsion et de doublage.

Mûrier : plante arborescente de la famille des Moracées, dont le fruit est la mûre et dont les feuilles servent à nourrir les vers à soie. II en existe deux espèces: le mûrier noir* et le mûrier blanc*.
Mûrier blanc (Morus alba, lin) : mûrier cultivé, à feuilles rondes, épaisses, abondantes et contenant beaucoup de suc. Il donne peu de fruits, mais ses feuilles conviennent bien aux vers à soie. Moins résistant que le mûrier noir, sur lequel il est souvent greffé pour l'élevage des vers à soie, il en existe néanmoins de nombreux cultivars.

Mûrier noir (Morus nigra, lin) : mûrier sauvage, à feuilles fines, pointues et contenant peu de suc, et qui donne des fruits en quantité. Sa racine solide et son tronc plein en font une espèce robuste. Les vers nourris de ses feuilles produisent une soie solide et élastique, appropriée au tissage de gaze. En raison de sa solidité, le mûrier noir sert souvent de support aux greffes.

Tirage de la soie : opération consistant à dissoudre partiellement le grès qui adhère aux brins de soie en trempant un nombre déterminé de cocons dans une bassine d'eau chaude à une température donnée. Par la suite, on tire les baves une à une par leur extrémité et on les fait passer par la filière* jusqu'au guindre* en passant par la croisure* et le va-et-vient. Grâce aux débris de grès restant sur la soie, les baves, au contact de l'air, adhérent entre elles pour former un seul fil. 